\title{
Editorials
}

\section{NICE guidelines series and the role of indicators}

\author{
NICK BAILLIE, ${ }^{1}$ NICOLA BENT, ${ }^{2}$ GILLIAN LENG,${ }^{3}$ TIM KENDALL,${ }^{4}$ BETH SHACKLETON,${ }^{5}$ \\ ${ }^{\prime}$ Programme Manager, Evidence and Uptake, NICE, National Institute for Health and \\ Clinical Excellence Implementation Directorate \\ ${ }^{2}$ Associate Director, Implementation Systems, NICE, National Institute for Health and \\ Clinical Excellence Implementation Directorate \\ ${ }^{3}$ Implementation Director, Deputy Chief Executive NICE, National Institute for Health \\ and Clinical Excellence Implementation Directorate \\ ${ }^{4}$ Joint Director, NCCMH, National Collaborating Centre for Mental Health \\ ${ }^{5}$ Guideline Implementation Advisor, NCCMH, National Collaborating Centre for Mental Health
}

\section{INTRODUCIION TO NICE}

The National Institute for Health and Clinical Excellence (NICE) is the independent organisation responsible for providing national guidance on the promotion of good health and the prevention and treatment of ill health for the NHS in England, Wales and Northern Ireland. The purpose of the organisation is to standardise care across the NHS and to eliminate variation in access to treatment. NICE provides guidance in three areas of health:

- Public health guidance addresses the promotion of good health and the prevention of ill health by the National Health Service (NHS), local authorities and the wider public and voluntary sector;

- Clinical guidelines address the appropriate treatment and care of people with specific diseases and conditions within the NHS;

- Health technology guidance addresses the use of new and existing medicines, treatments and procedures within the NHS. This work stream comprises technology appraisals and interventional procedures.

A wide range of guidance has been now published across a wide range of disease areas by NICE. The very

Address for correspondence: N. Bent, National Institute for Health and Clinical Excellence, MidCity Place 71, High Holborn, London WC1V 6NA (United Kindom).

E-mail: Nicola.Bent@nice.org.uk

Declaration of Interest: None. first clinical guideline developed under NICE processes addressed the treatment of schizophrenia (Institute of health and Clinical Excellence, 2002).

\section{THE NATIONAL COLLABORATING CENTRES}

NICE established seven National Collaborating Centres (NCCs) that drive the clinical guideline development programme. Most of the NCCs are based in the royal medical colleges and/or leading professional societies and can therefore harness the expertise of these institutions in developing the guidelines.

\section{GUIDELINE DEVELOPMENT IN MENTAL HEALTH}

The individual nature of mental health problems and their treatment, and the range and diversity of professionals and agencies involved in a person's care, might suggest that evidence-based guidelines drawn from a narrow base of effective interventions could not properly address the complexity of the problems. Moreover, the questionable validity of some diagnostic categories such as depression (Dohrenwend, 1990), and the substantial placebo effects in the treatment of many mental health problems (Walsh et al., 2002), might lead some to suggest that any claim to a proper evidence base for the treatment and care of any mental health is at best premature. While we accept that psychological disorders are likely to elude precise definition, and that mental health and social care is an amalgam of specific interventions, humanity

Epidemiologia e Psichiatria Sociale, 17, 4, 2008 
and compassion, our view is that rather than being a sterile distillation of barely relevant evidence, clinical guidelines can bring together the best evidence for treatment with the need for compassion and care.

Moreover, the purpose of clinical guidelines in mental health is to bring a high standard of care into the many different levels of service from psychiatry to social care and across the whole NHS and to reduce variation between services. Guidelines also disseminate research and professional consensus to service users and carers in order to raise awareness of mental health disorders and encourage active engagement with the services available.

The original Schizophrenia guideline was published in December 2002 and it is now the first clinical guideline in mental health to be updated. The National Collaborating Centre for Mental Health (NCCMH) is currently revising the guideline and the update shall be published in March 2009.

\section{The scope}

The guideline scope provides a framework and boundaries for guideline development. It is important to make sure that the size of the scope is manageable so that the guideline can be developed within 1 to 2 years, but not so narrow that the guideline would only be applicable to a small number of service users and cover a limited range of treatments. The scope must be limited to allow the completion of a guideline for as broad a group of people as can be managed within the development period. The precise coverage of the scope is agreed with a wide range of stakeholders through formal and informal consultation.

\section{Development}

At the same time as developing the scope, a Chair is appointed jointly by NICE and the NCCMH following a national advertisement. The Chair, in conjunction with a lead director and guideline facilitator from the NCCMH (Tim Kendall or Steve Pilling), appoints a representative guideline development group from a national field.

The multidisciplinary guideline development group (GDG), which includes, academic experts, clinicians, and service user and carer representatives then work together with the NCCMH technical team (comprising an information scientist, systematic reviewers, research assistants and health economists) following an internationally agreed guideline development process. There are five main steps in the guideline development process:
The GDG define clinical questions the guideline should answer either through evidence or consensus sufficiently to cover the guideline scope.

Guided by the clinical questions the NCCMH technical team and the GDG develop comprehensive search strategies of all relevant electronic databases (usually many thousands of studies are identified and collated at this stage).

Protocols that define the inclusion and exclusion criteria in terms of the quality of the studies and their relevancy are designed for evaluating the evidence identified.

Synthesis and (meta-) analysis are conducted on systematic reviews and/or randomised studies of high quality and the results are shown in forest plots, which are a graphic way of displaying the data and easy for all members of the GDG to interpret.

The clinical questions are then used to guide examination of the data and to generate evidence statements directly addressing, and answering where possible, the original clinical questions. To go from the evidence statements to recommendations about clinical practice, the GDG take a broad view of all the possible comparative outcomes for a treatment, including the likelihood of side effects and the acceptability of treatment.

In the original Schizophrenia guideline (National Institute of health and Clinical Excellence, 2002) recommendations were graded according to their evidential source.

Level A: Randomised controlled trials (RCT)

Level B: High quality evidence but not an RCT

Level C: Lacking good quality clinical studies so based on expert consensus

Good Practice Points (GPPs): Areas of practice where there is little evidence forthcoming or when value-based recommendations are deemed desirable by the GDG.

The feedback from implementing the original guideline showed that in practice clinicians would only look at the grade ' $A$ ' evidence even though some of the GPPs were of equal worth. NICE has therefore removed this grading structure from its guidelines.

When all the recommendations have been agreed by the GDG, a series of care pathways are developed tracing the routes and contexts within which service users and carers could receive treatment and help within the NHS. For most mental health guidelines this will include pathways through primary, community, secondary and tertiary care.

NICE has a strong commitment to rigour and transparency in its methods. In line with this, the guideline went through two rounds of formal consultation, 
although this has been modified and streamlined over the last few years. During consultation the first drafts of guideline products are submitted to NICE, placed on the NICE website, and sent to all registered stakeholders, special advisers and experts suggested by the GDG.

The response to the Schizophrenia guideline was very positive from professionals and patients alike. Formal reviews have also praised the guideline. The World Health Organization and the World Psychiatric Association carried out an evaluation of 24 national schizophrenia guidelines (Gaebel et al., 2005). The NICE guideline was ranked highest by a considerable margin on all but one of the chosen domains and was rated best overall: "We found a remarkable superiority of the NICE schizophrenia guideline". A review by Aymerich et al. (2004) came to similar conclusions. The Schizophrenia 'Information for Public' (IFP) summary booklet was recognised in its own right and was commended in the 2003 BMA medical book competition.

\section{NICE APPROACH TO IMPLEMENTATION}

Initially the role of NICE was solely to produce guidance for NHS organisations to implement. However it soon became apparent that implementation was not happening consistently across the NHS. NICE held a number of workshops to collate feedback on how it could support implementation and an implementation strategy was launched in 2004. The strategy is aimed at influencing the three levels outlined by Fixsen et al. (2005) individual behaviour, organisational processes and the external environment.

In order to do this the NICE implementation strategy has four key elements:

- raising awareness of the need to change

- motivating and inspiring people to change

- providing practical support to facilitate change

- evaluating and monitoring the impact of the strategy.

To operationalise this strategy NICE provides materials such as presenters slides to promote awareness; costing tools to facilitate local change projects; reports on the uptake of guidance to support benchmarking and works with key national partners to ensure guidance is embedded with professional, regulatory other support agencies and bodies. The full programme of support is detailed on the NICE website http://www.nice.org.uk/usingguidance/ and a number of the practical packages are freely available for download.

\section{ROLE OF INDICATORS}

One of the more recent additions to the NICE implementation programme, which is becoming increasingly important, is the development of clinical indicators and their incorporation into national and local data collection processes. The use of clinical indicators has proved to be important in the implementation programme as they are particularly flexible, can be constructed in a number of ways and are used for a variety of different purposes at a variety of different levels.

Firstly, indicators can be focussed on processes related to NICE guidance, for example the proportion of service users prescribed an atypical antipsychotic; and secondly they can relate to health outcomes, for example the proportion of service users with schizophrenia who rate their health as better following a period of treatment.

These two examples also demonstrate the flexibility of indicators in their ability to be constructed with both quantitative data and softer qualitative statements. They may also be constructed using a range of data sources whether it be patient surveys, clinical audit or research projects, routinely collected data in electronic information systems or organisations self-reporting against standards. The SIEPDIRECT'S Project (DIscrepancy between Routine practice and Evidence in psychiatric Community Treatments on Schizophrenia) (Ruggeri, 2008; Semisa et al., 2008; Ruggeri et al., 2008) presented in this issue clearly displays how indicators can be constructed in this way.

In the same way that indicators can be constructed in a multitude of ways, their role in implementation is wide ranging. Indicators can be used to understand the implementation and quality improvement process highlighting where progress has been made and where further effort is needed. Where indicators are used in more than one unit they can also allow comparisons to be made (benchmarking). Comparative indicators can again facilitate quality improvement, for example allowing organisations to learn from others whose indicators suggest practice is very positive. Finally indicators can be used to judge and assess performance.

In the NHS, indicators are used to highlight performance for a wide range of purposes but two of these are particularly topical in the current climate. Firstly, performance indicators are made publicly available to allow patients to make informed choices about where they receive their care. In England a NHS website has recently been launched providing patients with indicators relating to care at their local hospitals to enable them to choose where to receive care www.nhs.uk. Secondly indicators can be used in commissioning arrangements to monitor the delivery of services against contract specifications, but more specifically to provide financial incen-

Epidemiologia e Psichiatria Sociale, 17, 4, 2008 
tives or enhancements where certain levels are met. In the NHS there are specific examples of this including a scheme of payments to family doctors known as the Quality Outcomes Framework (QOF).

The final benefit of using indicators in the implementation process is that they can be used at any level. Individual practitioners can use indicators in relation to their own practice; teams, services and organisations can also use indicators for all the purposes outlined above and finally they can also be aggregated to regional or national level. Whilst indicators relating to the implementation of NICE guidance have not been used beyond this, there is no reason why with careful interpretation, they could not be used at an international scale to compare practice between Italian and British health systems and share learning such as the lessons gained from the SIEP-DIRECT'S Project.

\section{A CAUTIONARY NOTE ON INDICATORS}

Having highlighted the many benefits and advantages of using indicators in the implementation process, there are a number of limitations and cautions that must be described. Where routine data is available from electronic information systems the construction of indicators can be a relatively easy task but where other means of data collection are needed, such as surveys, audit and research projects they can become much more time intensive. This has not only implications for their construction in the first instance but also the longevity of the indicator. Making an indicator as easy to construct as possible means that it can be revaluated at routine intervals to demonstrate progress.

The data sources outlined in the SIEP-DIRECT'S Project shows the variety of information available.

Finally expertise in the construction of clinical indicators is particularly important to ensure that appropriate inclusions and exceptions to the nominator and denominators have been made. A period of testing as described in the SIEP-DIRECT'S Project and piloting indicators can provide great benefit and ensures there is uniformity in their interpretation, construction and data collection.

\section{CONCLUSIONS}

NICE and its NCCs have now developed one of the most ambitions and comprehensive evidence-based, independent guideline programmes internationally and soon will have covered 100 conditions. The first NICE guideline was for the treatment of Schizophrenia in primary and secondary care. This has since been used in many parts of the world and adopted in Australia, Italy and California. Soon NICE and the NCCMH will have developed 20 guidelines in mental health covering all the main psychoses, substance misuse problems, common mental disorders, personality disorders, childhood mental health and the dementias.

While continuing to update all these guidelines overtime, NICE and the NCCMH are now addressing more clearly some of the more difficult and pressing problems facing physical and mental health but also using the NICE methodology to address other areas such as social care and education. NICE and the NCCMH have also become much more focussed on changing practice and ensuring that NICE guidelines are woven into the fabric of clinical care. To this end the implementation directorate, the NCCs, and the NHS more widely, are facing up to the challenge of guidelines. At 60 years old, and with the imminent expansion of the NICE guidelines programme, a new NHS based upon the best evidence and values of high-quality practice is ever more likely to become a reality. The extension of NICE and the NCCMH's work into other countries and areas like the SIEP-DIRECT'S Project presented in the current monographic issue of EPS can only be welcomed.

\section{REFERENCES}

Aymerich M., Guillamon I., San Emerterio M., Teixido M., Sanchez I., Faus G., Lalucat L. \& Matinez C. (2004). Clinical practice guidelines in schizophrenia: How to select the best one? Schizophrenia Research 67, Suppl. 1, 139.

Dohrenwend B.P. (1990). The problem of validity in field studies of psychological disorders' revisited. Psychological Medicine 20, 195-208.

Fixsen D., Naoom S., Blase K., Friedman R. \& Wallace F. (2005). Implementation Research: A Synthesis of the Literature. University of South Florida: Florida.

Gaebel W., Weinmann S., Sartorius N., Rutz W. \& McIntyre J.S. (2005). Schizophrenia practice guidelines: international survey and comparison. British Journal of Psychiatry 187, 248-255.

National Institute of Health and Clinical Excellence (2002). Schizophrenia: Full National Clinical Guideline on Core Interventions in Primary and Secondary Care. Retrieved July 14, 2008, from http://www.nice.org.uk/nicemedia/pdf/cg001fullguideline.

Ruggeri M. (2008). Guidelines for treating mental illness: love them, hate them. Can the SIEP-DIRECT'S Project serve in the search for a happy medium? Epidemiologia e Psichiatria Sociale 17(4), 270-277.

Ruggeri M., Lora A. \& Semisa D. on behalf of the SIEP-DIRECT'S Group (2008). The SIEP-DIRECT'S Project on the discrepancy between routine practice and evidence. An outline of the main findings and the practical implications for the future of community based mental health services Epidemiologia e Psichiatria Sociale 17(4), 358-367.

Semisa D., Lora A., Morosini P. \& Ruggeri M. (2008). The SIEPDIRECT'S Project on the discrepancy between routine practice and evidence in the treatment of schizophrenia. The design, the indicators, and the methodology of the study (in Italian). Epidemiologia e Psichiatria Sociale 17(4), 278-290.

Walsh T., Seidman S., Sysko R. \& Gould M. (2002). Placebo response in studies of major depression: variable, substantial, and growing. Journal of American Medical Association 287, 1840-1847. 